\title{
Modified Dispersion Relations and Black Hole Entropy
}

\author{
Remo Garattini* \\ Università degli Studi di Bergamo, Facoltà di Ingegneria, \\ Viale Marconi 5, 24044 Dalmine (Bergamo) ITALY. and \\ INFN - sezione di Milano, Via Celoria 16, Milan, Italy.
}

\begin{abstract}
We compute the black hole entropy in the context of the Modified Dispersion Relations using the brick wall model. An explicit dependence of the radial coordinate approaching the horizon is shown to analyze the behavior of the divergence. We find that, due to the modification of the density of states, the brick wall can be eliminated. By assuming a specific form for the radial coordinate $r\left(E / E_{P}\right)$, we examine a possible candidate for $r\left(E / E_{P}\right)$. A comparison with the 't Hooft approach is presented.
\end{abstract}

\section{INTRODUCTION}

After almost thirty years after the introduction of the famous Bekenstein-Hawking formula [1, 2]

$$
S_{B H}=\frac{1}{4} A / l_{P}^{2}
$$

relating the entropy of a black hole and its area, the thermodynamics of such objects still attracts research in this direction. One reason is due to the lack of a Quantum Gravity theory which should be able to explain black hole physics. Another reason comes from the fact that Hawking radiation 2] develops modes of arbitrarily high frequency near the horizon. The appearance of a trans-Planckian physics in Black Hole thermodynamics has led many authors to consider that some deep change in particle physics should come into play. In connection to this idea, in recent years, there has been a proposal on how the fundamental aspects of special relativity can be modified at very high energies. This modification has been termed Doubly Special Relativity (DSR) [3]. In DSR, the Planck mass is regarded as an observer independent energy scale. This assumption has as effect in momentum space that the usual dispersion relation for a massive particle of mass $m$ is changed into the following expression

$$
E^{2} g_{1}^{2}\left(E / E_{P}\right)-p^{2} g_{2}^{2}\left(E / E_{P}\right)=m^{2},
$$

where $g_{1}\left(E / E_{P}\right)$ and $g_{2}\left(E / E_{P}\right)$ are two functions which have the following property

$$
\lim _{E / E_{P} \rightarrow 0} g_{1}\left(E / E_{P}\right)=1 \quad \text { and } \quad \lim _{E / E_{P} \rightarrow 0} g_{2}\left(E / E_{P}\right)=1 .
$$

The usual dispersion relation is recovered at low energies. Eqs.(23) are a representation of "Modified Dispersion Relations" (MDRs). The common motivation in using them is in that they can be used as a phenomenological approach to investigate physics at the Planck scale, where General Relativity is no longer reliable. Concerning Black Hole thermodynamics, it has been proved that the spectrum emitted at infinite distance from the hole is only marginally affected by MDRs [5]. Nevertheless, when we consider the statistical thermodynamics of quantum fields in the Hartle-Hawking state (i.e. having the Hawking temperature $T_{H}$ at large radii), to keep under control the high frequency divergences coming from the horizon sector, we need some kind of cut-off of Planckian size known as "brick wall" [6]. In a series of papers, it has been suggested that this divergence could be absorbed in a renormalization of Newton's constant [7 [9], while other authors approached the problem of the divergent brick wall using Pauli-Villars regularization [10 12]. Another interesting proposal comes from non-commutative geometry which introduces a natural thickness of the horizon replacing the 't Hooft's brick wall 17]. Other successful attempts come by the modification of the Heisenberg uncertainty relations, known as Generalized Uncertainty Principle (GUP) [13 [16]. The modified inequality takes the form

$$
\Delta x \Delta p \geq \hbar+\frac{\lambda_{p}^{2}}{\hbar}(\Delta p)^{2}
$$

*Electronic address: Remo.Garattini@unibg.it 
where $\hbar$ is the Planck constant and $\lambda_{p}$ is the Planck length. The interesting point regards exactly the modified number of quantum states, which is changed into

$$
\frac{d^{3} x d^{3} p}{(2 \pi \hbar)^{3}\left(1+\lambda p^{2}\right)^{3}}
$$

When $\lambda=0$, the formula reduces to the ordinary counting of quantum states. If Eq.(5) is used for computing the entropy, the brick wall can be removed [13, 14]. Note that GUP and MDRs modifications are strictly connected[18]. This suggest that we could use MDRs to remove the brick wall. MDRs have a deep impact also when the background is curved. Indeed, the analysis of Magueijo and Smolin 19] shows that the energy-momentum tensor and the Einstein equations are replaced by a one parameter family of equations

$$
G_{\mu \nu}(E)=8 \pi G(E) T_{\mu \nu}(E)+g_{\mu \nu} \Lambda(E),
$$

where $G(E)$ is an energy dependent Newton's constant, defined so that $G(0)$ is the physical Newton's constant. Similarly we have an energy dependent cosmological constant $\Lambda(E)$. In this context, the modified Friedmann-RobertsonWalker line element changes into 19]

$$
d s^{2}(E)=-\frac{d t^{2}}{g_{1}^{2}(E)}+\frac{a^{2}(t)}{g_{2}^{2}(E)} g_{i j} d x^{i} d x^{j},
$$

where $g_{i j}$ represents the spatially homogeneous and isotropic metric of a sphere (positive curvature $K=1$ ), pseudosphere (with negative curvature $K=-1$ ), or euclidean space $\left(K=0\right.$, so that $g_{i j}=\delta_{i j}$ ). Note that the metric coefficients are energy dependent. When a gravitational background has a structure like the one in the line element (7), we have a "rainbow metric". When the Schwarzschild line is examined, the related rainbow metric reads

$$
d s^{2}(E)=-\left(1-\frac{2 M G(0)}{r}\right) \frac{d \tilde{t}^{2}}{g_{1}^{2}(E)}+\frac{d \tilde{r}^{2}}{\left(1-\frac{2 M G(0)}{r}\right) g_{2}^{2}(E)}+\frac{\tilde{r}^{2}}{g_{2}^{2}(E)}\left(d \theta^{2}+\sin ^{2} \theta d \phi^{2}\right) .
$$

We expect the functions $g_{1}(E)$ and $g_{2}(E)$ modify the UV behavior in the same way as GUP and Noncommutative geometry do, respectively. Since the form of $g_{1}(E)$ and $g_{2}(E)$ is unknown and they have to obey the property (3), we have a large amount of arbitrariness in fixing the dependence on $E / E_{P}$, even if some specific choices have been proposed by G. Amelino-Camelia et al. 4, 20] in the context of black hole thermodynamics. The rest of the paper is structured as follows, in section [1] we compute the free energy and we examine its contribution near the horizon, in section [II we compute the relevant thermodynamical quantities. We summarize and conclude in section IV] Units in which $\hbar=c=k=1$ are used throughout the paper.

\section{W.K.B. APPROACH WITH MDRS}

Instead of working with the background of form 7 or 8 we adopt the following general aspect for the line element, useful for spherically symmetric problems [21]

$$
d s^{2}=-\exp (-2 \Lambda(r))\left(1-\frac{b(r)}{r}\right) \frac{d t^{2}}{g_{1}^{2}\left(E / E_{P}\right)}+\frac{d r^{2}}{\left(1-\frac{b(r)}{r}\right) g_{2}^{2}\left(E / E_{P}\right)}+\frac{r^{2}}{g_{2}^{2}\left(E / E_{P}\right)} d \Omega^{2} .
$$

Usually, this kind of metric is adopted for the description of wormholes. However, it is quite general to include as special cases the Schwarzschild, Reissner-Nordström and de Sitter and Anti-de Sitter geometries, or any combination of these. The function $b(r)$ will be referred to as the "shape function". The shape function may be thought of as specifying the shape of the spatial slices. On the other hand, $\Lambda(r)$ will be referred to as the "redshift function" that describes how far the total gravitational redshift deviates from that implied by the shape function. Without loss of generality we can fix the value of $\Lambda(r)$ at infinity such that $\Lambda(\infty)=0$. If the equation $b\left(r_{w}\right)=r_{w}$ is satisfied for some values of $r$, then we say that the points $r_{w}$ are horizons for the metric (9). For the outermost horizon one has $\forall r>r_{w}$ that $b(r)<r$. Consequently $b^{\prime}\left(r_{w}\right) \leq 1$. We will fix our attention to the $b^{\prime}\left(r_{w}\right)<1$ case only. The anomalous case $b^{\prime}\left(r_{w}\right)=1$ can be thought as describing extreme black holes where an inner and outer horizons are merged and will not be considered here. For a spherically symmetric system the surface gravity is computed via

$$
\kappa_{w}=\lim _{r \rightarrow r_{w}}\left\{\frac{1}{2} \frac{\partial_{r} g_{t t}}{\sqrt{g_{t t} g_{r r}}}\right\}
$$


and for the metric (9), we get

$$
\kappa_{w}=\lim _{r \rightarrow r_{w}} \frac{1}{2}\left\{\frac{\exp (-\Lambda(r))}{h\left(E / E_{P}\right)}\left[-2 \Lambda^{\prime}(r)\left(1-\frac{b(r)}{r}\right)+\frac{b(r)}{r^{2}}-\frac{b^{\prime}(r)}{r}\right]\right\},
$$

with

$$
h\left(E / E_{P}\right)=\frac{g_{1}\left(E / E_{P}\right)}{g_{2}\left(E / E_{P}\right)} .
$$

By assuming that $\Lambda\left(r_{w}\right)$ and $\Lambda^{\prime}\left(r_{w}\right)$ are both finite we obtain that

$$
\kappa_{w}=\frac{1}{2 r_{w} h(E)} \exp \left(-\Lambda\left(r_{w}\right)\right)\left[1-b^{\prime}\left(r_{w}\right)\right],
$$

where, in the proximity of the throat we have approximated $1-b(r) / r$ with

$$
1-\frac{b(r)}{r}=\frac{r-r_{w}}{r_{w}}\left[1-b^{\prime}\left(r_{w}\right)\right]
$$

Now that the geometrical framework has been set up, we begin with a real massless scalar field described by the action $^{1}$

$$
I=-\frac{1}{2} \int d^{4} x \sqrt{-g}\left[g^{\mu \nu} \partial_{\mu} \phi \partial_{\nu} \phi\right]
$$

in the background geometry of Eq.(9) whose Euler-Lagrange equations are

$$
\frac{1}{\sqrt{-g}} \partial_{\mu}\left(\sqrt{-g} g^{\mu \nu} \partial_{\nu}\right) \phi=0
$$

If $\phi$ has the separable form

$$
\phi(t, r, \theta, \varphi)=\exp (-i E t) Y_{l m}(\theta, \varphi) f(r)
$$

then the equation for $f(r)$ reads

$$
\left[\frac{g_{2}^{2}(E) \exp (\Lambda(r))}{r^{2}} \partial_{r}\left(r^{2} \exp (-\Lambda(r))\left(1-\frac{b(r)}{r}\right) \partial_{r}\right)-\frac{l(l+1)}{r^{2}}+\frac{E_{n l}^{2} g_{1}^{2}(E) \exp (2 \Lambda(r))}{1-\frac{b(r)}{r}}\right] f_{n l}=0,
$$

where $Y_{l m}(\theta, \varphi)$ is the usual spherical harmonic function. In order to make our system finite let us suppose that two mirror-like boundaries are placed at $r=r_{1}$ and $r=R$ with $R \gg r_{1}, r_{1}>r_{w}$ and consider Dirichlet boundary conditions $f_{n l}\left(r_{1}\right)=f_{n l}(R)=0$. We also assume the set of real functions $\left\{f_{n l}(r)\right\}(n=1,2, \cdots)$, defined by Eq.(18), be complete with respect to the space of $L_{2}$-functions on the interval $r_{1} \leq r \leq R$ for each $l$. The positive constant $\omega_{n l}$ is defined as the corresponding eigenvalue. In order to use the WKB approximation, we define an r-dependent radial wave number $k(r, l, E)$

$$
k_{r}^{2}(r, l, E) \equiv \frac{1}{\left(1-\frac{b(r)}{r}\right)}\left[\exp (2 \Lambda(r)) \frac{E^{2} h^{2}(E)}{\left(1-\frac{b(r)}{r}\right)}-\frac{l(l+1)}{r^{2}}\right]
$$

where we have used Eq.(12). The number of modes with frequency less than $E$ is given approximately by

$$
\tilde{g}(E)=\int_{0}^{l_{\max }} \nu(l, E)(2 l+1) d l
$$

\footnotetext{
${ }^{1}$ See also Ref. [22] for a derivation of the brick wall in the Boulware state.
} 
where $\nu(l, E)$ is the number of nodes in the mode with $(l, E)$ :

$$
\nu(l, E)=\frac{1}{\pi} \int_{r_{w}}^{R} \sqrt{k^{2}(r, l, E)} d r .
$$

Here it is understood that the integration with respect to $r$ and $l$ is taken over those values which satisfy $r_{w} \leq r \leq R$ and $k^{2}(r, l, E) \geq 0$. Thus, from Eq.(19) we get

$$
\begin{aligned}
& \frac{d \tilde{g}(E)}{d E}=\int \frac{\partial \nu(l, E)}{\partial E}(2 l+1) d l=\frac{1}{\pi} \int_{r_{w}}^{R} d r \int_{0}^{l_{\max }} d l(2 l+1) \frac{\exp (2 \Lambda(r))}{\sqrt{k^{2}(r, l, E)}} \frac{\left(E h^{2}(E)+E^{2} h(E) h^{\prime}(E)\right)}{\left(1-\frac{b(r)}{r}\right)^{2}} \\
= & \frac{2}{\pi} E h(E)\left(E h^{2}(E)+E^{2} h(E) h^{\prime}(E)\right) \int_{r_{w}}^{R} d r \frac{\exp (3 \Lambda(r))}{\left(1-\frac{b(r)}{r}\right)^{2}} r^{2}=\frac{2}{\pi} \frac{d}{d E}\left(\frac{1}{3} E^{3} h^{3}(E)\right) \int_{r_{w}}^{R} d r \frac{\exp (3 \Lambda(r))}{\left(1-\frac{b(r)}{r}\right)^{2}} r^{2} .
\end{aligned}
$$

The free energy is given approximately by

$$
F=\frac{1}{\beta} \int_{0}^{\infty} \ln \left(1-e^{-\beta E}\right) \frac{d \tilde{g}(E)}{d E} d E,
$$

where $\beta$ is the inverse temperature measured at infinity. Then from Eq.(22), we find

$$
\begin{aligned}
F= & \frac{2}{\pi} \frac{1}{\beta} \int_{0}^{\infty} \ln \left(1-e^{-\beta E}\right) d E E^{2} h^{2}(E)\left(h(E)+E h^{\prime}(E)\right) \int_{r_{w}}^{R} d r r^{2} \frac{\exp (3 \Lambda(r))}{\left(1-\frac{b(r)}{r}\right)^{2}} \\
& =\frac{2}{\pi} \frac{1}{\beta} \int_{0}^{\infty} \ln \left(1-e^{-\beta E}\right) \frac{d}{d E}\left(\frac{1}{3} E^{3} h^{3}(E)\right) d E \int_{r_{w}}^{R} d r r^{2} \frac{\exp (3 \Lambda(r))}{\left(1-\frac{b(r)}{r}\right)^{2}} .
\end{aligned}
$$

It is convenient to divide the free energy into two pieces denoted by

$$
F_{r_{w}}=\frac{2}{\pi} \frac{1}{\beta} \int_{0}^{\infty} \ln \left(1-e^{-\beta E}\right) d E E^{2} h^{2}(E)\left(h(E)+E h^{\prime}(E)\right) \int_{r_{w}}^{r_{1}} d r r^{2} \frac{\exp (3 \Lambda(r))}{\left(1-\frac{b(r)}{r}\right)^{2}}
$$

and

$$
F_{R}=\frac{2}{\pi} \frac{1}{\beta} \int_{0}^{\infty} \ln \left(1-e^{-\beta E}\right) d E E^{2} h^{2}(E)\left(h(E)+E h^{\prime}(E)\right) \int_{r_{1}}^{R} d r r^{2} \frac{\exp (3 \Lambda(r))}{\left(1-\frac{b(r)}{r}\right)^{2}}
$$

Assuming that $\Lambda(r)<\infty, \forall r \in\left[r_{w},+\infty\right), F_{R}$ is dominated by large volume effects for large $R$. This is particular evident in the case when $b(r)<r$. Indeed, we get for the radial dependent integral

$$
F_{R} \sim \frac{2}{\pi \beta} \frac{R^{3}}{3} \int_{0}^{\infty} \ln \left(1-e^{-\beta E}\right) \frac{d}{d E}\left(\frac{1}{3} E^{3} h^{3}(E)\right) d E .
$$

This term will give the contribution to the entropy of a homogeneous quantum gas in flat space at a uniform temperature $T$ when a MDR of the form (2) is considered. We will not examine this large volume contribution here. To study $F_{r_{w}}$, we use Eq.(14), then the radial part of $F_{r_{w}}$ becomes divergent in proximity of $r_{w}$. This ultraviolet divergence has been cured by 't Hooft, who introduced a "brick wall $r_{0}$ " proportional to $l_{P}^{2}$. This is obtained by keeping only the leading divergence in $F_{r_{w}}$ and introducing the proper distance from the throat

$$
\alpha=\int_{r_{w}}^{r_{w}+r_{0}} \frac{d r}{\sqrt{1-\frac{b(r)}{r}}}=\frac{2 \sqrt{r_{0}}}{\sqrt{\frac{1-b^{\prime}\left(r_{w}\right)}{r_{w}}}} .
$$


Then $F_{r_{w}}$ becomes

$$
\int_{r_{w}+r_{0}}^{r_{1}} d r r^{2} \frac{\exp (3 \Lambda(r))}{\left(1-\frac{b(r)}{r}\right)^{2}} \simeq 4 r_{w}^{5} \frac{\exp \left(3 \Lambda\left(r_{w}\right)\right)}{\left(1-b^{\prime}\left(r_{w}\right)\right)^{3}} \frac{1}{\alpha^{2}} \simeq 4 r_{w}^{5} \frac{\exp \left(3 \Lambda\left(r_{w}\right)\right)}{\left(1-b^{\prime}\left(r_{w}\right)\right)^{3}} \frac{1}{l_{P}^{2}}
$$

Nevertheless, since spacetime is modified by a "rainbow metric", it is quite natural that even the "brick wall" is affected by this distortion. To see such an effect, we perform the radial integration in $F_{r_{w}}$, to obtain

$$
\int_{r_{w}+r_{0}}^{r_{1}} d r r^{2} \frac{\exp (3 \Lambda(r))}{\left(1-\frac{b(r)}{r}\right)^{2}}=\int_{r_{w}+r\left(E / E_{P}\right)}^{r_{1}} d r r^{2} \frac{\exp (3 \Lambda(r))}{\left(1-\frac{b(r)}{r}\right)^{2}} \simeq r_{w}^{4} \frac{\exp \left(3 \Lambda\left(r_{w}\right)\right)}{\left(1-b^{\prime}\left(r_{w}\right)\right)^{2}} \frac{1}{r\left(E / E_{P}\right)}
$$

where we have assumed that, in proximity of the throat the brick wall is no longer a constant but it becomes a function of $E / E_{P}{ }^{2}$. Plugging Eq.(31) into $F_{r_{w}}$, we obtain

$$
F_{r_{w}}=\frac{2 r_{w}^{4}}{\pi \beta} \frac{\exp \left(3 \Lambda\left(r_{w}\right)\right)}{\left(1-b^{\prime}\left(r_{w}\right)\right)^{2}} \int_{0}^{\infty} \frac{\ln (1-\exp (-\beta E))}{r\left(E / E_{P}\right)} \frac{d}{d E}\left(\frac{1}{3} E^{3} h^{3}\left(E / E_{P}\right)\right) d E .
$$

After an integration by parts, one gets

$$
F_{r_{w}}=-\frac{C_{r_{w}}}{3 \beta} \int_{0}^{\infty} E^{3} h^{3}\left(E / E_{P}\right) \frac{d}{d E}\left[\frac{\ln (1-\exp (-\beta E))}{r\left(E / E_{P}\right)}\right] d E,
$$

where $h\left(E / E_{P}\right)$ is chosen in such a way to allow the convergence when $E / E_{P} \rightarrow \infty$ and

$$
C_{r_{w}}=\frac{2 r_{w}^{4}}{\pi} \frac{\exp \left(3 \Lambda\left(r_{w}\right)\right)}{\left(1-b^{\prime}\left(r_{w}\right)\right)^{2}} .
$$

Since one is interested to an elimination of the "brick wall" when $E / E_{P} \rightarrow 0$ we assume, without loss of generality, that

$$
r\left(E / E_{P}\right)=r_{w} \sigma\left(E / E_{P}\right)
$$

with

$$
\sigma\left(E / E_{P}\right) \rightarrow 0, \quad E / E_{P} \rightarrow 0
$$

Plugging Eq.(37) into Eq.(35), we obtain

$$
\begin{gathered}
F_{r_{w}}=-\frac{C_{r_{w}}}{3 \beta r_{w}} \int_{0}^{\infty} E^{3} h^{3}(E) \frac{d}{d E}\left[\frac{\ln (1-\exp (-\beta E))}{\sigma\left(E / E_{P}\right)}\right] d E \\
=-\frac{C_{r_{w}}}{3 \beta r_{w}} \int_{0}^{\infty} \frac{E^{3} h^{3}(E)}{\sigma\left(E / E_{P}\right)}\left[\frac{\beta}{(\exp (\beta E)-1)}-\frac{\ln (1-\exp (-\beta E))}{E_{P} \sigma\left(E / E_{P}\right)} \sigma^{\prime}\left(E / E_{P}\right)\right] d E,
\end{gathered}
$$

where the prime means that we are computing the derivative with respect to the argument. We can see what happens to the free energy $F_{r_{w}}$ for some specific forms of $g_{1}\left(E / E_{P}\right)$ and $g_{2}\left(E / E_{P}\right)$. One popular choice is given by

$$
g_{1}\left(E / E_{P}\right)=1-\eta\left(E / E_{P}\right)^{n} \quad \text { and } \quad g_{2}\left(E / E_{P}\right)=1
$$

${ }^{2}$ In Ref. 23], it has been introduced a radial dependent cut-off by setting

$$
\sqrt{1-\frac{r_{w}}{r_{E}}\left(1-b^{\prime}\left(r_{w}\right)\right) \Lambda}=E .
$$

This leads to

$$
r_{E}=r_{w} \frac{\left(1-b^{\prime}\left(r_{w}\right)\right) \Lambda^{2}}{\Lambda^{2}\left(1-b^{\prime}\left(r_{w}\right)\right)-E^{2}} \quad \rightarrow \quad r_{w}
$$

when $\Lambda \rightarrow \infty$. Thus, the rôle of $\Lambda$ is analogous to the brick wall. 
where $\eta$ is a dimensionless parameter and $n$ is an integer [24]. Thus the form of $h\left(E / E_{P}\right)$ is

$$
h\left(E / E_{P}\right)=1-\eta\left(E / E_{P}\right)^{n} .
$$

Nevertheless, the above choice does not allow the integration by parts in Eq. 34 and therefore will be discarded. Thus the choice of the possible forms of $g_{1}\left(E / E_{P}\right)$ and $g_{2}\left(E / E_{P}\right)$ is strongly restricted by convergence criteria and by the property (38). One good candidate for the convergence is

$$
h\left(E / E_{P}\right)=\exp \left(-\frac{E}{E_{P}}\right) .
$$

In section 【II, we will discuss some choices for $\sigma\left(E / E_{P}\right)$.

\section{ENTROPY AND TOTAL ENERGY WITH MDRS}

The property described in (38) shows that the divergence on the throat is represented by $\sigma\left(E / E_{P}\right)$. However, the distortion due to the rainbow metric in Eq.(40) introduces a term $E^{3}$ which partially cures the throat divergence. As an illustrative example, we consider the following form for

$$
\sigma\left(E / E_{P}\right)=h^{\delta}\left(E / E_{P}\right)\left(\frac{E}{E_{P}}\right)^{\alpha}
$$

which, of course do not exhaust the possible candidates for MDRs. We have two interesting cases:

a) $\delta=0 ; \alpha>0$

and

b) $\delta>0 ; \alpha>0$.

With choice a) Eq.(35) becomes

$$
F_{r_{w}}=-\frac{C_{r_{w}} E_{P}^{\alpha}}{3 \beta r_{w}} \int_{0}^{\infty} E^{3} \exp \left(-3 E / E_{P}\right)\left[\frac{\beta}{(\exp (\beta E)-1) E^{\alpha}}-\frac{\alpha \ln (1-\exp (-\beta E))}{E^{\alpha+1}}\right] d E .
$$

The second integral considerably simplifies for $\alpha=2$. From the Appendix , we find

$$
F_{r_{w}}=-\frac{2 C_{r_{w}} E_{P}^{2}}{3 \beta^{2} r_{w}}\left[\zeta\left(2,1+\frac{3}{\beta E_{P}}\right)+\frac{\beta E_{P}}{3}\left(\gamma+\Psi\left(1+\frac{3}{\beta E_{P}}\right)\right)\right],
$$

where $\zeta(s, \nu)$ is the Hurwitz zeta function, $\Gamma(x)$ is the gamma function and $\Psi(x)$ is the digamma function. Since the Hurwitz zeta function obeys the relation

$$
\frac{d}{d \nu} \zeta(s, \nu)=-s \zeta(s+1, \nu)
$$

we can easily compute the other relevant thermodynamic quantities. The total energy $U$ is defined by

$$
\begin{aligned}
U & =\frac{\partial\left(\beta F_{r_{w}}\right)}{\partial \beta}=-\frac{2 C_{r_{w}} E_{P}^{2}}{3 r_{w}} \frac{\partial}{\partial \beta}\left[\frac{1}{\beta} \zeta\left(2,1+\frac{3}{\beta E_{P}}\right)+\frac{E_{P}}{3}\left(\gamma+\Psi\left(1+\frac{3}{\beta E_{P}}\right)\right)\right] \\
& =\frac{2 C_{r_{w}} E_{P}^{2}}{3 r_{w} \beta^{2}}\left[\zeta\left(2,1+\frac{3}{\beta E_{P}}\right)-\zeta\left(3,1+\frac{3}{\beta E_{P}}\right)\left(\frac{6}{\beta E_{P}}\right)+\Psi^{\prime}\left(1+\frac{3}{\beta E_{P}}\right)\right]
\end{aligned}
$$

and the entropy $S$ is

$$
S=\beta^{2} \frac{\partial F_{r_{w}}}{\partial \beta}=-\beta^{2} \frac{2 C_{r_{w}} E_{P}^{2}}{3 r_{w}} \frac{\partial}{\partial \beta}\left[\frac{1}{\beta^{2}} \zeta\left(2,1+\frac{3}{\beta E_{P}}\right)+\frac{E_{P}}{3 \beta}\left(\gamma+\Psi\left(1+\frac{3}{\beta E_{P}}\right)\right)\right]
$$




$$
\begin{gathered}
=\frac{2 C_{r_{w}} E_{P}^{2}}{3 r_{w}}\left[\frac{2}{\beta} \zeta\left(2,1+\frac{3}{\beta E_{P}}\right)+\frac{E_{P}}{3}\left(\gamma+\Psi\left(1+\frac{3}{\beta E_{P}}\right)\right)\right. \\
\left.\quad-\zeta\left(3,1+\frac{3}{\beta E_{P}}\right)\left(\frac{6}{\beta^{2} E_{P}}\right)+\frac{1}{\beta} \Psi^{\prime}\left(1+\frac{3}{\beta E_{P}}\right)\right] .
\end{gathered}
$$

In the limit where $\beta E_{P} \gg 1$, we can take the leading order of the total energy $U$

$$
U=\frac{2 C_{r_{w}} E_{P}^{2}}{3 \beta^{2} r_{w}}\left[\zeta(2)+\frac{\pi^{2}}{6}\right]=\frac{2 C_{r_{w}} E_{P}^{2}}{3 \beta^{2} r_{w}} \frac{\pi^{2}}{3}=r_{w}^{3} \frac{\exp \left(3 \Lambda\left(r_{w}\right)\right)}{\left(1-b^{\prime}\left(r_{w}\right)\right)^{2}} \frac{4 E_{P}^{2}}{9 \beta^{2}} \pi
$$

and the entropy $S$

$$
S=\frac{2 C_{r_{w}} E_{P}^{2}}{3 r_{w}}\left[\frac{2}{\beta} \zeta(2)+\frac{E_{P}}{3}\left(\gamma-\gamma+\frac{\pi^{2}}{2 \beta E_{P}}\right)+\frac{\pi^{2}}{6 \beta}\right]=r_{w}^{3} \frac{\exp \left(3 \Lambda\left(r_{w}\right)\right)}{\left(1-b^{\prime}\left(r_{w}\right)\right)^{2}} \frac{8 E_{P}^{2}}{9 \beta} \pi,
$$

where we have used Eq.(36). Moreover, recalling the expression for the surface gravity in the low energy limit, we get

$$
\kappa_{w}=\frac{1}{2 r_{w}} \exp \left(-\Lambda\left(r_{w}\right)\right)\left[1-b^{\prime}\left(r_{w}\right)\right] .
$$

then $U$

$$
U=r_{w}^{2} \frac{\exp \left(2 \Lambda\left(r_{w}\right)\right)}{1-b^{\prime}\left(r_{w}\right)} \frac{2 E_{P}^{2}}{9 \beta^{2} \kappa_{w}} \pi
$$

and the entropy $S$

$$
S=r_{w}^{2} \frac{\exp \left(2 \Lambda\left(r_{w}\right)\right)}{1-b^{\prime}\left(r_{w}\right)} \frac{4 E_{p}^{2}}{9 \beta \kappa_{w}} \pi
$$

lead to

$$
U=r_{w}^{2} \frac{\exp \left(2 \Lambda\left(r_{w}\right)\right)}{1-b^{\prime}\left(r_{w}\right)} \frac{E_{P}^{2}}{9 \beta}
$$

and the entropy $S$

$$
S=\frac{A_{r_{w}} E_{P}^{2}}{4} \frac{\exp \left(2 \Lambda\left(r_{w}\right)\right)}{1-b^{\prime}\left(r_{w}\right)} \frac{2}{9 \pi}
$$

where we have used

$$
\frac{1}{\beta}=T=\frac{\kappa_{w}}{2 \pi}
$$

To recover the area law, we have to set

$$
\frac{\exp \left(2 \Lambda\left(r_{w}\right)\right)}{1-b^{\prime}\left(r_{w}\right)}=\frac{9 \pi}{2} .
$$

This corresponds to a changing of the time variable with respect to the Schwarzschild time. The total energy becomes

$$
U=r_{w}^{2} \frac{\pi E_{P}^{2}}{2 \beta}
$$

which in terms of the Schwarzschild radius $r_{w}=2 M G$ and inverse Hawking temperature $\beta=8 \pi M G$ becomes

$$
U=4 M^{2} G^{2} \frac{E_{P}^{2}}{16 M G}=\frac{M}{4} .
$$

Note the discrepancy of a factor of $3 / 2$ with the 't Hooft result. 
With the choice $\mathbf{b}), F_{r_{w}}$ becomes

$$
\begin{gathered}
F_{r_{w}}=-\frac{C_{r_{w}}}{3 \beta r_{w}} \int_{0}^{\infty} d E E^{3} h^{3}(E)\left[\frac{E_{P}^{\alpha} \beta}{(\exp (\beta E)-1) h^{\delta}(E) E^{\alpha}}\right. \\
\left.-\frac{E_{P}^{\alpha} \ln (1-\exp (-\beta E))}{h^{\delta}\left(E / E_{P}\right) E^{\alpha} E_{P}}\left(\delta E_{P} \frac{h^{\prime}\left(E / E_{P}\right)}{h\left(E / E_{P}\right)}+\alpha \frac{E_{P}}{E}\right)\right] .
\end{gathered}
$$

Since $h(E)$ assumes the form 43, we can further simplify the above integral

$$
F_{r_{w}}=-\frac{C_{r_{w}} E_{P}^{\alpha}}{3 \beta r_{w}} \int_{0}^{\infty} d E E^{3-\alpha} h^{3-\delta}(E)\left[\frac{\beta}{(\exp (\beta E)-1)}-\ln (1-\exp (-\beta E))\left(-\frac{\delta}{E_{P}}+\frac{\alpha}{E}\right)\right] .
$$

Fixing $\alpha=2$ and letting $\delta$ unspecified, we get

$$
F_{r_{w}}=-\frac{C_{r_{w}} E_{P}^{2}}{3 \beta r_{w}}\left\{\frac{2}{\beta} \zeta\left(2,1+\frac{3-\delta}{\beta E_{P}}\right)+\frac{\delta E_{P}}{(3-\delta)^{2}}\left[\gamma+\Psi\left(1+\frac{3-\delta}{\beta E_{P}}\right)\right]+\delta \frac{\Psi^{\prime}\left(1+\frac{3-\delta}{\beta E_{P}}\right)}{\beta(3-\delta)}+\frac{\pi^{2}}{3 \beta}\right\} .
$$

where we have integrated by parts the last term of Eq.(62). The value of $\delta=3$ has to be treated as a separate case, which reduces $F_{r_{w}}$ to

$$
F_{r_{w}}=-\frac{C_{r_{w}} E_{P}^{2}}{3 \beta r_{w}} \int_{0}^{\infty} d E E\left[\frac{\beta}{(\exp (\beta E)-1)}+3 \frac{\ln (1-\exp (-\beta E))}{E_{P}}-2 \frac{\ln (1-\exp (-\beta E))}{E}\right] .
$$

After an integration by parts on the second and third term, we obtain

$$
F_{r_{w}}=-\frac{C_{r_{w}} E_{P}^{2}}{3 \beta^{2} r_{w}} \int_{0}^{\infty} d x\left[\frac{3 x^{2}}{2 \beta E_{P}\left(e^{x}-1\right)}+\frac{3 x}{\left(e^{x}-1\right)}\right]=-\frac{C_{r_{w}} E_{P}^{2}}{\beta^{2} r_{w}}\left(\frac{\pi^{2}}{6}+\frac{\zeta(3)}{\beta E_{P}}\right) .
$$

With the help of Eq.(52) and in the approximation $\beta E_{P} \gg 1$, the relevant thermodynamical quantities are

$$
U=\frac{C_{r_{w}} E_{P}^{2}}{6 \beta^{2} r_{w}} \pi^{2}=r_{w}^{2} \frac{\exp \left(2 \Lambda\left(r_{w}\right)\right)}{1-b^{\prime}\left(r_{w}\right)} \frac{E_{P}^{2}}{12 \beta}
$$

and

$$
S=\frac{\pi^{2} C_{r_{w}} E_{P}^{2}}{3 \beta r_{w}}=\frac{A_{r_{w}}}{4} E_{P}^{2} \frac{\exp \left(2 \Lambda\left(r_{w}\right)\right)}{1-b^{\prime}\left(r_{w}\right)} \frac{1}{6 \pi} .
$$

Even in this case, to recover the area law, we have to set

$$
\frac{\exp \left(2 \Lambda\left(r_{w}\right)\right)}{1-b^{\prime}\left(r_{w}\right)}=6 \pi
$$

and the total energy becomes

$$
U=\frac{C_{r_{w}} E_{P}^{2}}{6 \beta^{2} r_{w}} \pi^{2}=r_{w}^{2} \frac{\pi E_{P}^{2}}{2 \beta} .=\frac{M}{4} .
$$

In terms of the inverse Hawking temperature and of the Schwarzschild radius, we get

$$
U=\frac{M}{4}
$$

differing from the 't Hooft result by a factor of 3/2. When $\delta \neq 3$, from Eq. (B2) of Appendix B, we extract the form of the internal energy

$$
U=r_{w}^{2} \frac{\exp \left(2 \Lambda\left(r_{w}\right)\right)}{1-b^{\prime}\left(r_{w}\right)} \frac{E_{P}^{2}}{6 \beta}\left[\frac{\delta}{3-\delta}+\frac{2}{3}\right]
$$


while for the entropy from Eq.(B5), we can write

$$
S=r_{w}^{2} \frac{\exp \left(2 \Lambda\left(r_{w}\right)\right)}{1-b^{\prime}\left(r_{w}\right)} \frac{E_{P}^{2}}{3}\left[\frac{\delta}{6(3-\delta)}+\frac{2}{3}\right]=\frac{A_{r_{w}}}{4} E_{P}^{2} \frac{\exp \left(2 \Lambda\left(r_{w}\right)\right)}{1-b^{\prime}\left(r_{w}\right)} \frac{1}{3 \pi}\left[\frac{\delta}{6(3-\delta)}+\frac{2}{3}\right] .
$$

If we set

$$
\frac{\exp \left(2 \Lambda\left(r_{w}\right)\right)}{1-b^{\prime}\left(r_{w}\right)}=9 \pi\left[\frac{\delta}{2(3-\delta)}+2\right]^{-1}
$$

we recover the area law and the internal energy becomes

$$
U=r_{w}^{2} \pi \frac{E_{P}^{2}}{\beta}\left[\frac{\delta+6}{12-\delta}\right] .
$$

\section{SUMMARY AND DISCUSSION}

In this paper, we have examined the possibility that MDRs alter space time so deeply that even the black hole physics close to the horizon is affected. In particular, we have examined the possibility that MDRs cure the high frequency pathology known as brick wall. We have based our approach using a particular form of MDRs known as "gravity's rainbow" which introduces two unknown functions $g_{1}\left(E / E_{P}\right)$ and $g_{2}\left(E / E_{P}\right)$ in the background metric. These two unknown functions have the property (3) which means that for low energy, we are dealing with ordinary gravity. Since we are interested in the entropy and energy computation respectively, we have used the WKB approximation which introduces a density of states dependent on $g_{1}\left(E / E_{P}\right)$ and $g_{2}\left(E / E_{P}\right)$. Although the form of the "rainbow" functions $g_{1}\left(E / E_{P}\right)$ and $g_{2}\left(E / E_{P}\right)$ is undetermined, we can receive hints from the function $h\left(E / E_{P}\right)$ in Eq. (12), representing the ratio between $g_{1}\left(E / E_{P}\right)$ and $g_{2}\left(E / E_{P}\right)$. Indeed, it appears that in order to have finite values of the free energy, $h\left(E / E_{P}\right)$ must be of a form to allow the convergence of the integral in Eq.(35). Note that the "rainbow metric" (3) affects the free energy UV by means of the term $\frac{1}{3} E^{3} h^{3}\left(E / E_{P}\right)$ in the density of states and plays a central role in the elimination of the brick wall. A key point comes from the assumption that the brick wall can be energy dependent. Unfortunately, an explicit expression of such a dependence cannot be extracted at this level. It is likely that such an expression be revealed by a further examination of Einstein's field equations in the context of a "gravity's rainbow". A tentative of computing a form of $r\left(E / E_{P}\right)$ has been suggested in Ref.[23], even if a sharp UV cutoff has been used . If one is tempted to perform a series expansion at low energies

$$
r\left(E / E_{P}\right)=r^{\prime}\left(E / E_{P}\right) \frac{E}{E_{P}}+\ldots
$$

with the condition

$$
r\left(E / E_{P}\right) \rightarrow 0 \quad \text { when } \quad E / E_{P} \rightarrow 0,
$$

one discovers form Eq.(40) that the result is finite. However, this works only for a low energy limit. Therefore, it appears to be advantageous to guess what families of functions can be used to have a finite free energy and therefore the related entropy. Indeed, the choice (44) represents a good combination of functions having the right properties for the elimination of the brick wall. We wish to remark that the disappearance of the brick wall is due to the presence of MDRs and not to the fact that $r \equiv r\left(E / E_{P}\right)$ close to the horizon. Of course one can choose a dependence on $E$ such that the divergence disappears without invoking MDRs. Nevertheless, MDRs are a consequence of a space time modification, which affect even the horizon behavior. In a sense the simple modification of $r \rightarrow r\left(E / E_{P}\right)$ represents the quantum fluctuations of the throat. That a modification of space time be a cure of divergences in entropy computation has been shown with a specific model of space time foam [25] context, where the degree of the divergence has been lowered to logarithmic [26]. This result suggests that space time foam be in strong connection with MDRs. How to explicitly realize such a connection will be the subject of a further investigation [27].

\section{Appendix A: Integrals}

In this Appendix, we explicitly compute the integrals appearing in Eq.(45). We begin with

$$
\int_{0}^{\infty} d E \frac{h^{\delta}(E) E^{3-\alpha}}{e^{\beta E}-1}=\int_{0}^{\infty} d E \frac{\exp \left(-\delta E / E_{P}\right) E^{3-\alpha}}{e^{\beta E}-1} .
$$


By setting $\beta E=x$, we get

$$
\int_{0}^{\infty} d x \frac{\exp \left[-\left(1+\delta /\left(\beta E_{P}\right)\right) x\right] x^{3-\alpha}}{1-e^{-x}}=\frac{\Gamma(4-\alpha)}{\beta^{4-\alpha}} \zeta\left(4-\alpha, 1+\frac{\delta}{\beta E_{P}}\right),
$$

where we have used the formula

$$
\int_{0}^{\infty} d x \frac{x^{\nu-1} \exp (-\mu x)}{1-e^{-\beta x}}=\frac{\Gamma(\nu)}{\beta^{\nu}} \zeta\left(\nu, \frac{\mu}{\beta}\right) \quad \begin{aligned}
& \operatorname{Re} \mu>0 \\
& \operatorname{Re} \nu>1
\end{aligned} .
$$

The term

$$
\int_{0}^{\infty} d E E h^{3-\delta}(E) \ln \left(1-e^{-\beta E}\right)=\int_{0}^{\infty} d E E \exp \left(-(3-\delta) E / E_{P}\right) \ln \left(1-e^{-\beta E}\right)
$$

can be cast into the form $(\beta E=x)$

$$
\begin{gathered}
=\frac{1}{\beta^{2}} \int_{0}^{\infty} d x x \exp \left(-\frac{(3-\delta) x}{\beta E_{P}}\right) \ln \left(1-e^{-x}\right)=-\frac{d}{d \mu}\left[\frac{1}{\beta^{2}} \int_{0}^{\infty} d x \exp \left(-\left(\mu+\frac{(3-\delta)}{\beta E_{P}}\right) x\right) \ln \left(1-e^{-x}\right)\right]_{\mid \mu=0} \\
\quad=-\frac{d}{d \mu}\left[\frac{1}{\beta^{2}} \int_{0}^{\infty} d x \exp (-(a+\mu) x) \ln \left(1-e^{-x}\right)\right]_{\mid \mu=0}=-\frac{d}{d \mu}\left[\frac{1}{\beta^{2}} \int_{0}^{1} d t(1-t)^{a+\mu-1} \ln (t)\right]_{\mid \mu=0}
\end{gathered}
$$

with

$$
a=\frac{3-\delta}{\beta E_{P}}
$$

The result of the integration gives

$$
\begin{gathered}
\int_{0}^{1} d t(1-t)^{a+\mu-1} \ln (t)=B(1, a+\mu)[\Psi(1)-\Psi(1+a+\mu)] \\
=\frac{\Gamma(a+\mu)}{\Gamma(1+a+\mu)}[\Psi(1)-\Psi(1+a+\mu)]=\frac{1}{a+\mu}[-\gamma-\Psi(1+a+\mu)],
\end{gathered}
$$

where $B(x, y)$ is the Beta function, $\Psi(x)$ is the digamma function and where we have used the property

$$
\Gamma(1+x)=x \Gamma(x) .
$$

The integral A2 becomes

$$
-\frac{d}{d \mu}\left[\frac{1}{\beta^{2}}\left(\frac{1}{a+\mu}[-\gamma-\Psi(1+a+\mu)]\right)\right]_{\mid \mu=0}=\frac{1}{\beta^{2}}\left[\left(\frac{1}{a^{2}}[\gamma+\Psi(1+a)]\right)+\frac{\Psi^{\prime}(1+a)}{a}\right],
$$

where we have used the relation

$$
\int_{0}^{1} d x x^{\mu-1}\left(1-x^{r}\right)^{\nu-1} \ln (x)=\frac{1}{r^{2}} B\left(\frac{\mu}{r}, \nu\right)\left[\Psi\left(\frac{\mu}{r}\right)-\Psi\left(\frac{\mu}{r}+\nu\right)\right] \quad \begin{gathered}
\operatorname{Re} \mu \\
\operatorname{Re} \nu \\
r>0
\end{gathered}
$$

\section{Appendix B: Case $\delta \neq 3$}

In this Appendix, we compute the internal energy $U$ and the entropy $S$ for $\delta \neq 3$. For the internal energy, we obtain

$$
U=\frac{\partial\left(\beta F_{r_{w}}\right)}{\partial \beta}=\frac{C_{r_{w}} E_{P}^{2}}{3 \beta^{2} r_{w}}\left[2 \zeta\left(2,1+\frac{3-\delta}{\beta E_{P}}\right)+\delta \frac{\Psi^{\prime}\left(1+\frac{3-\delta}{\beta E_{P}}\right)}{3-\delta}+\frac{\pi^{2}}{3}\right]
$$




$$
+\frac{C_{r_{w}} E_{P}^{2}}{3 \beta r_{w}}\left(\frac{3-\delta}{\beta^{2} E_{P}}\right)\left\{-4 \zeta\left(2,1+\frac{3-\delta}{\beta E_{P}}\right)+\frac{\delta E_{P}}{(3-\delta)^{2}} \Psi^{\prime}\left(1+\frac{3-\delta}{\beta E_{P}}\right)+\delta \frac{\Psi^{\prime \prime}\left(1+\frac{3-\delta}{\beta E_{P}}\right)}{(3-\delta)}\right\} .
$$

In the limit $\beta E_{P} \gg 1$, we find

$$
U \simeq \frac{C_{r_{w}} E_{P}^{2}}{3 \beta^{2} r_{w}}\left[2 \zeta(2)+\delta \frac{\Psi^{\prime}(1)}{3-\delta}+\frac{\pi^{2}}{3}\right]=\frac{C_{r_{w}} E_{P}^{2}}{3 \beta^{2} r_{w}} \pi^{2}\left[\frac{\delta}{3-\delta}+\frac{2}{3}\right] .
$$

For the entropy, from the definition one gets

$$
\begin{gathered}
F_{r_{w}}=-\frac{C_{r_{w}} E_{P}^{2}}{3 \beta r_{w}}\left\{\frac{2}{\beta} \zeta\left(2,1+\frac{3-\delta}{\beta E_{P}}\right)+\frac{\delta E_{P}}{(3-\delta)^{2}}\left[\gamma+\Psi\left(1+\frac{3-\delta}{\beta E_{P}}\right)\right]+\delta \frac{\Psi^{\prime}\left(1+\frac{3-\delta}{\beta E_{P}}\right)}{\beta(3-\delta)}+\frac{\pi^{2}}{3 \beta}\right\} . \\
S=\beta^{2} \frac{\partial F_{r_{w}}}{\partial \beta}=\frac{C_{r_{w}} E_{P}^{2}}{3 r_{w}}\left[\frac{4}{\beta} \zeta\left(2,1+\frac{3-\delta}{\beta E_{P}}\right)+2 \delta \frac{\Psi^{\prime}\left(1+\frac{3-\delta}{\beta E_{P}}\right)}{\beta(3-\delta)}+\frac{2 \pi^{2}}{3 \beta}+\frac{\delta E_{P}}{(3-\delta)^{2}}\left[\gamma+\Psi\left(1+\frac{3-\delta}{\beta E_{P}}\right)\right]\right] \\
+\frac{C_{r_{w}} E_{P}}{3 \beta r_{w}}(3-\delta)\left\{-\frac{4}{\beta} \zeta\left(3,1+\frac{3-\delta}{\beta E_{P}}\right)+\frac{\delta E_{P}}{(3-\delta)^{2}} \Psi^{\prime}\left(1+\frac{3-\delta}{\beta E_{P}}\right)+\delta \frac{\Psi^{\prime \prime}\left(1+\frac{3-\delta}{\beta E_{P}}\right)}{\beta(3-\delta)}\right\} .
\end{gathered}
$$

Always in the same limit, we can approximate the entropy as

$$
S \simeq \frac{2 C_{r_{w}} E_{P}^{2}}{3 r_{w} \beta} \pi^{2}\left[\frac{\delta}{6(3-\delta)}+\frac{2}{3}\right] .
$$

\section{Appendix C: Acknowledgments}

The author would like to thank S. Liberati and G. Amelino-Camelia for useful discussions and suggestions.

[1] J. D. Bekenstein, Phys. Rev. D 7, 949 (1973).

[2] S. W. Hawking, Comm. Math. Phys. 43, 199 (1975).

[3] G. Amelino-Camelia, Int.J.Mod.Phys. D 11, 35 (2002); gr-qc/0012051 G. Amelino-Camelia, Phys.Lett. B 510, 255 (2001); hep-th/0012238.

[4] G. Amelino-Camelia, Phys. Lett. B 510, 255 (2001); hep-th/0012238. G. Amelino-Camelia and T. Piran, Phys. Rev. D 64, 036005 (2001); astro-ph/0008107 T. Jacobson, S. Liberati and D. Mattingly, Phys. Rev. D 66, 081302 (2002); hep-ph/0112207. G. Amelino-Camelia, Mod. Phys. Lett. A 17, 899 (2002); gr-qc/0204051. S. Sarkar, Mod. Phys. Lett. A 17, 1025 (2002); gr-qc/0204092 T. J. Konopka and S. A. Major, New J. Phys. 4, 57 (2002); hep-ph/0201184. J. Alfaro and G. Palma, Phys. Rev. D 67, 083003 (2003); hep-th/0208193. G. Amelino-Camelia, Int. J. Mod. Phys. D 11, 1643 (2002); gr-qc/0210063 D. Heyman, F. Hinteleitner and S. Major, Phys. Rev. D 69, 105016 (2004); gr-qc/0312089. T. A. Jacobson, S. Liberati, D. Mattingly and F. W. Stecker, Phys. Rev. Lett. 93, 021101 (2004); astro-ph/0309681. J. Magueijo and L. Smolin, Phys. Rev. Lett. 88, 190403 (2002); hep-th/0112090. J. Magueijo and L. Smolin, Phys. Rev. D 67, 044017 (2003); gr-qc/0207085. D. V. Ahluwalia-Khalilova, Int. J. Mod. Phys. D 13, 335 (2004); gr-qc/0402023 J. R. Ellis, N. E. Mavromatos, D. V. Nanopoulos, A. S. Sakharov and E. K. G. Sarkisyan, Astropart. Phys. 25 (2006) 402; astro-ph/0510172.

[5] W. G. Unruh, Phys. Rev. Lett. 46, 1351 (1981). S. Hossenfelder, Phys.Lett. B 649, 310 (2007); gr-qc/0612167.

[6] G. 't Hooft, Nucl. Phys. B 256, 727 (1985).

[7] L. Susskind and J. Uglum, Phys. Rev. D 50, 2700 (1994).

[8] J.L.F. Barbon and R. Emparan, Phys. Rev. D 52, 4527 (1995), hep-th/9502155.

[9] E. Winstanley, Phys. Rev. D 63, 084013 (2001), hep-th/0011176

[10] J.- G. Demers, R. Lafrance and R.C. Myers, Phys. Rev. D 52, 2245 (1995), gr-qc/9503003.

[11] D. V. Fursaev and S. N. Solodukhin, Phys. Lett. B 365, 51 (1996), hep-th/9412020

[12] S. P. Kim, S. K. Kim, K.-S. Soh and Jae Hyung Yee, Int. J. Mod. Phys. A 12, 5223 (1997), gr-qc/9607019.

[13] X. Li, Phys. Lett. B 540, 9 (2002), gr-qc/0204029 
[14] Z. Ren, W. Yue-Qin and Z. Li-Chun, Class. Quant. Grav. 20 (2003), 4885.

[15] G. Amelino-Camelia, Class.Quant.Grav. 23, 2585 (2006), gr-qc/0506110. G. Amelino-Camelia, Gen.Rel.Grav. 33, 2101 (2001), gr-qc/0106080.

[16] Consequences of this modification have been discussed in A. Kempf, G. Mangano and R.B. Mann, Phys. Rev. D 52, 1108 (1995), hep-th/9412167, L. J. Garay, Int. J. Mod. Phys. A 10, 145 (1995), gr-qc/9403008 D. V. Ahluwalia, Phys. Lett. A 275, 31 (2000), gr-qc/0002005; R. J. Adler, P. Chen and D. I. Santiago, Gen. Rel. Grav. 33, 2101 (2001), gr-qc/0106080 S.K. Rama, Phys. Lett. B 519, 103 (2001), hep-th/0107255 L. N. Chang, D. Minic, N. Okamura and Tatsu Takeuchi, Phys. Rev. D 65, 125028 (2002), hep-th/0201017.

[17] H. Bai and M. L. Yan, JHEP 758 (2003), gr-qc/0303006.

[18] J. L. Cortes and J. Gamboa, Phys. Rev. D 71, 065015 (2005); hep-th/0405285

[19] J. Magueijo and L. Smolin, Class. Quant. Grav. 21, 1725 (2004) arXiv:gr-qc/0305055.

[20] G. Amelino-Camelia, M. Arzano, Y. Ling and G. Mandanici, Class. Quant. Grav. 23, 2585 (2006); gr-qc/0506110

[21] M. Visser, Lorentzian Wormholes (AIP Press, New York, 1995) 64.

[22] S. Mukohyama and W. Israel, Phys. Rev. D 58, 104005 (1998), gr-qc/9806012

[23] L. Randall, V. Sanz and M. D. Schwartz, JHEP 8, 0206 (2002), hep-th/0204038

[24] Y. Ling, JCAP 17, 708 (2007), gr-qc/0609129. Y. Ling, X. Li and H. Zhang, Mod.Phys.Lett. A 22, 2749 (2007), gr-qc/0512084, Y. Ling, B. Hu and X. Li, Phys. Rev. D 73, 087702 (2006), gr-qc/0512083

[25] R. Garattini, Int. J. Mod. Phys. D 4, 635 (2002), gr-qc/0003090.

[26] R. Garattini, Mod. Phys. Lett. A 19, 2673 (2004), gr-qc/0409015

[27] R. Garattini, in preparation. 